\title{
TALEN-induced disruption of Nanog expression results in reduced proliferation, invasiveness and migration, increased chemosensitivity and reversal of EMT in HepG2 cells
}

\author{
AI QING YU ${ }^{1,2^{*}}, Y_{A N}$ DING ${ }^{1 *}$, CHENG LIN LI $^{1}$, YI YANG ${ }^{1}$, SHI RONG YAN $^{1-3}$ and DONG SHENG LI ${ }^{1}$ \\ ${ }^{1}$ Hubei Key Laboratory of Embryonic Stem Cell Research, Taihe Hospital, Hubei University of Medicine, \\ Shiyan, Hubei 442000; ${ }^{2}$ Center for Gene Diagnosis, Zhongnan Hospital of Wuhan University, Wuhan, Hubei 430071; \\ ${ }^{3}$ Department of Biochemistry, Hubei University of Medicine, Shiyan, Hubei 442000, P.R. China
}

Received September 30, 2015; Accepted November 5, 2015

DOI: $10.3892 /$ or.2015.4483

\begin{abstract}
Accumulating evidence indicates that Nanog plays a central role in modulating the biological behaviors of human hepatocellular carcinoma (HCC). However, the underlying mechanisms remain unclear. In the present study, we employed transcription activator-like effector nucleases (TALEN) to disrupt Nanog expression in HepG2 cells and obtained subcloned cells with diallelic Nanog mutations. Significantly, we found that the expression of pluripotency factors Sox2, Oct4 and Klf4, as well as expression of cancer stem cell (CSC) marker CD133, in the Nanog-targeted HepG2 cells was markedly downregulated. This finding suggests that Nanog may play an important role in maintaining the pluripotency and malignancy of HepG2 cells. We also revealed that Nanog regulated cell proliferation by modulating the expression of cyclin D1/D3/E1 and CDK2, respectively. Additionally, the disruption of Nanog resulted in the downregulation of epithelial-mesenchymal transition (EMT) regulators Snail
\end{abstract}

Correspondence to: Professor Dong Sheng Li, Hubei Key Laboratory of Embryonic Stem Cell Research, Taihe Hospital, Hubei University of Medicine, Shiyan, Hubei 442000, P.R. China

E-mail: dsli1698@126.com

Professor Shi Rong Yan, Department of Biochemistry, Hubei University of Medicine, Shiyan, Hubei 442000, P.R. China

E-mail: graceyan@163.com

${ }^{*}$ Contributed equally

Abbreviations: TALEN, transcription activator-like effector nucleases; CSCs, cancer stem cells; HCC, hepatocellular carcinoma; EMT, epithelial-mesenchymal transition; ESCs, embryonic stem cells; CDK2, cyclin-dependent kinase 2; MMP2, matrix metallopeptidase 2; MDR1, multi-drug resistant gene 1; ABCG2, ATP-binding cassette subfamily G member 2; T7E1, T7 endonuclease 1

Key words: TALEN, Nanog, Oct4, Sox2, EMT, HepG2 cells, human hepatocellular carcinoma and Twist, which contributed to the elevated level of epithelial marker E-cadherin, and to the decreased level of mesenchymal markers $\mathrm{N}$-cadherin and vimentin in the HepG2 cells. In addition, the Nanog-targeted HepG2 cells exhibited reduced ability of invasion, migration and chemoresistance in vitro. In conclusion, the disruption of Nanog expression results in less proliferation, invasiveness, migration, more chemosensitivity and reversal of EMT in HepG2 cells, by which Nanog plays crucial roles in influencing the malignant phenotype of HepG2 cells.

\section{Introduction}

Human hepatocellular carcinoma (HCC) is considered as the third leading cause of cancer-related death worldwide (1-3). In recent years, although surgical treatment has greatly improved the survival rate of $\mathrm{HCC}$ patients, its prognosis and survival rate remain poor due to frequent intra-hepatic and extrahepatic metastases and dissemination $(3,4)$. Consequently, further investigation of the underlying molecular mechanisms are vital to identify novel therapeutic interventions and to improve the prognosis for $\mathrm{HCC}$.

Nanog, a central transcription regulator required for maintaining the self-renewal capacity and pluripotent state of embryonic stem cells (ESCs) along with Oct4 and Sox 2 (5-10), exhibits elevated expression in various types of tumor cells $(11,12)$. Intensive studies indicate that Nanog also executes parallel functions as in ESCs, participating in tumorigenesis and promoting the progression of certain types of tumors, including pancreatic cancer, gastrointestinal tumors, breast cancer, head and neck squamous cell carcinomas and human HCC (13-16). In addition, our previous study was the first to demonstrate that Nanog is involved in the invasion, chemoresistance, clonogenicity, migration and metastasis of the cervical carcinoma HeLa cell line (17). Nevertheless, the precise role of Nanog in human HCC has not been fully explored.

Epithelial-mesenchymal transition (EMT), which was initially identified as a characteristic of morphogenesis during embryogenesis, is a transdifferentiation program that reprograms adherent epithelial cells into more phenotypical 
mesenchymal cells. During EMT, epithelial cancer cells exhibit downregulation of the epithelial marker E-cadherin, but upregulation of mesenchymal markers N-cadherin and vimentin, promoting the acquisition of mesenchymal traits that are required for migration and invasion $(2,18,19)$. Developmental genetics studies have revealed that the orchestration of EMT is critical to the development of malignant traits, such as motility, invasiveness, metastasis, dissemination and apoptosis-resistance in various tumor cells (20-22). However, emerging molecular mechanisms and the signalingnetwork underlying the regulatory relationship between Nanog and the EMT pathway in the HCC HepG2 cell line remain unclear.

TALEN is gene editing tools with high efficiency and specificity and with low genotoxicity in targeted genome manipulation. TALEN is composed of repeats of DNA-binding domains and the FokI nuclease domain. TALEN-mediated double-strand breaks (DSBs) promote endogenous DNA repair mechanisms through error-prone non-homologous endjoining (NHEJ), by which TALEN successfully cleave the target genome and induce the mutation of target genes (23-25). Compared with RNA interference technology, TALEN offers the advantage of achieving robust disruption of target gene expression (26).

In the present study, we employed TALEN to induce diallelic Nanog mutations to disrupt Nanog expression in HepG2 cells. Most significantly, we demonstrated that the disruption of Nanog expression resulted in reduced proliferation, invasiveness, migration, enhanced chemosensitivity and reversal of EMT in HepG2 cells. Thereby, Nanog plays an important role in retaining the malignant phenotype of HepG 2 cells.

\section{Materials and methods}

Cell lines and culture. The human HCC cell line HepG2 was cultured in Dulbecco's modified Eagle's medium (DMEM) containing $10 \%$ fetal bovine serum (FBS) (both from Gibco ${ }^{\circledR}$ Life Technologies, USA) under a humidified condition at $37^{\circ} \mathrm{C}$ with $5 \% \mathrm{CO}_{2}$.

TALEN plasmid construction and Nanog targeting. The Nanog-TALEN plasmid was constructed according to our previous study (17) using a Fast TALE ${ }^{\mathrm{TM}}$ TALEN Assembly kit (SiDan-Sai Biotechnology, China). Approximately $10 \mu \mathrm{g}$ of the Nanog-TALEN plasmid (5 $\mu$ g each for right and left arm) and control plasmid were mixed with wild-type HepG2 cells $\left(1 \times 10^{6}\right)$ in $90 \mu \mathrm{l}$ of Opti-MEM (Gibco ${ }^{\circledR}$ Life Technologies), and were transferred to electroporation cuvettes for electroblotting. After the cells were transfected with Nanog-TALEN and the control plasmid under $150 \mathrm{~V}$, the cells were exposed to $3 \mu \mathrm{g} /$ $\mathrm{ml}$ puromycin for 3 days. Then, the medium containing puromycin was replaced with growth media.

T7 endonuclease 1 (T7E1) and genomic sequencing. Genomes derived from the Nanog-targeted and control cells were extracted using a Genomic DNA Extraction Mini kit (Tiangen, China). The DNA fragment surrounding the Nanog-targeted site was amplified and then analyzed by T7E1 (View Solid Biotechnology, China) to evaluate Nanog-mutation efficiency. The Nanog mutation was confirmed by sequencing.
Cell proliferation assays. The proliferation of targeted and control cells was monitored using an RTCA SP system, which is a real-time cell-based assay system (ACEA Biosciences, San Diego, CA, USA). According to the manufacturer's instructions, $5 \times 10^{3}$ cells were seeded in triplicate in an E-Plate VIEW 16 for real-time monitoring of the proliferative capacities of the cells for $120 \mathrm{~h}$.

Cell migration assays. Scratch assays were used to determine the migratory ability of HepG2 cells in compliance with our previous description (17). The cells were maintained in triplicate into 6-well plates until completely confluent, and then a scratch was created in the confluent cell monolayers using a $10-\mu 1$ pipette tip. After the cells were washed 3 times with phosphate-buffered saline (PBS), the cells was cultured in serum-free DMEM. The migratory capacity was assessed at $48 \mathrm{~h}$ under a microscope.

Matrigel invasion assays. We employed a Transwell invasion assay (17) to measure the invasive abilities of the cells in vitro. Cells were trypsinized and resuspended in DMEM at a density of $2 \times 10^{5}$ cells $/ \mathrm{ml}$. Approximately $200 \mu \mathrm{l}$ of cell suspension was added in triplicate to the upper chamber of the polycarbonate membrane filter, which was embedded with $8-\mu$ m pores (Corning, USA), that were pre-coated with Matrigel. The lower chamber was filled with $500 \mu$ l DMEM supplemented with $10 \%$ FBS. After the cells were incubated for $48 \mathrm{~h}$, non-migrating cells on the surface of the Matrigel in the upper chamber were wiped off by cotton swabs, whereas the cells migrating to the bottom of the membrane were fixed in $95 \%$ ethanol for $30 \mathrm{~min}$, and then stained with $0.1 \%$ crystal violet. After the stained membrane was washed 3 times with PBS, it was observed under a microscope and the number of migrating cells was counted.

Chemosensitivity assays. Chemosensitivity assays were performed to evaluate the sensitivity of Nanog-targeted and control cells to chemotherapeutics. Trypsinized cells were washed with PBS and then seeded in triplicate in an E-Plate VIEW 16 at a density of $1 \times 10^{4}$ cells/well for real-time monitoring using an RTCA SP system. After $6 \mathrm{~h}$, cisplatin (40 $\mu \mathrm{g} / \mathrm{ml}$ ) was added to the E-Plate VIEW 16 for detection of chemosensitivity at $72 \mathrm{~h}$.

Quantitative PCR. Total RNA from the Nanog-targeted and control cells was extracted using TRIzol reagent (Invitrogen, USA). The corresponding cDNA was synthesized using a reverse transcription kit (Takara, China). SYBR-Green PCR Master Mix was utilized to amplify the corresponding genes of interest. The PCR cycling program was as follows: $95^{\circ} \mathrm{C}$ for $5 \mathrm{~min}$, followed by 32 cycles of $95^{\circ} \mathrm{C}$ for $30 \mathrm{sec}, 58^{\circ} \mathrm{C}$ for $30 \mathrm{sec}, 72^{\circ} \mathrm{C}$ for $30 \mathrm{sec}$ with a final 10 -min incubation at $72^{\circ} \mathrm{C}$. The relative levels of gene expression were analyzed by the $2^{-\Delta \Delta \mathrm{Ct}}$ method. The fold-change of mRNA was normalized to $\beta$-actin. Primer sets for corresponding genes were the same as in our previous study (17).

Western blot analysis. Nanog-targeted and control cells were collected, washed with PBS; and then lysed on ice using protein lysis buffer and protein inhibitors. Protein lysates were mixed 


\section{A Nanog gene}

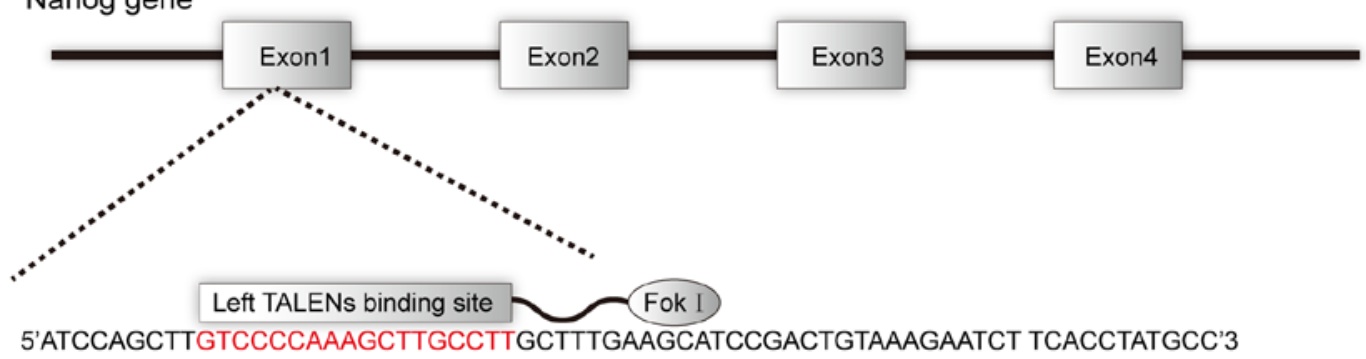

3'TAGGTCGAACAGGGGTTTCGAACGGAACGAAACTTCGTAGGCTGACATTTCTTAGAAGAGGATACGG'5

Fok I Right TALENs binding site

B

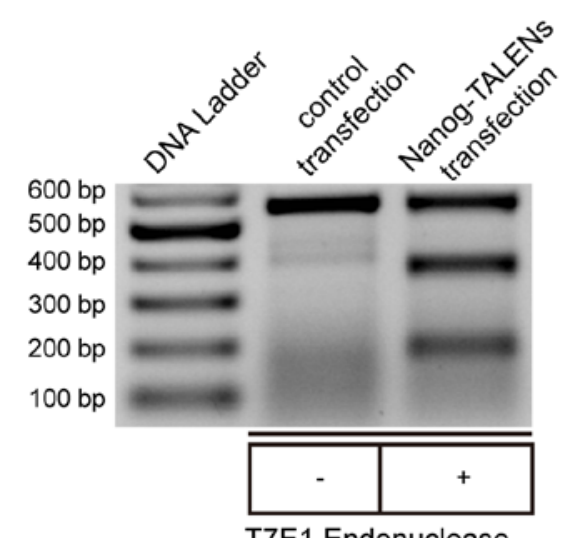

C
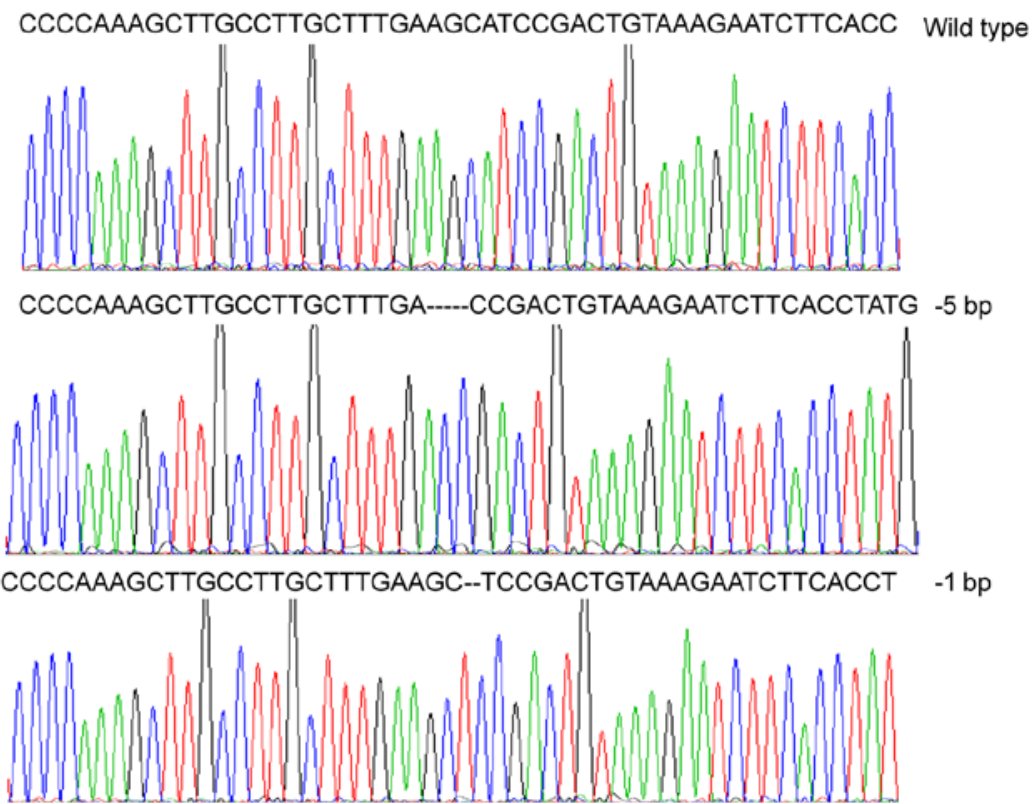

Figure 1. TALEN-mediated Nanog disruption. (A) Schematic representation of the Nanog locus and Nanog-TALEN design. (B) T7E1 endonuclease analysis of the efficiency of genomic Nanog mutation in the Nanog-targeted mixed HepG2 cells. (C) Representative genomic sequencing results of the Nanog mutation around the target site in the subclone 7 . The black dotted line represents the nucleotide deletion and the number of the nucleotide deletion is indicated on the right.

with SDS-PAGE protein loading buffer (5:1) and boiled for 5 min, followed by separation by $12 \%$ SDS-PAGE. Then, the proteins of interest were transferred onto polyvinylidene fluoride (PVDF) membranes (Millipore, USA). After the membranes were blocked in $5 \%$ non-fat milk in tris-buffered saline with Tween-20 (TBST), they were incubated overnight at $4^{\circ} \mathrm{C}$ with primary antibodies, respectively: anti-Nanog (no. sc-374103), anti-Twist (no. sc-81417) (1:500; Santa Cruz Biotechnology, USA), anti-Sox 2 (no. D6D9), anti-Oct4 (no. 2750) (1:500; Cell Signaling, USA), anti-CD133 (no. bs-4770R), anti-CDK2 (no. bs-10726R), anti-cyclin E (no bs-0573), anti-E-cadherin (no bs.1519R), anti-N-cadherin (no. bs-1172R), anti-vimentin (no. bs-0756R), anti-ABCG2 (no. bs-0662R), anti-MDR1 (no.bs-0563R) (1:400; Bioss, China), anti-cyclin D1 (no. AC853), anti-cyclin D3 (no AC856), anti-GAPDH (no. AG019) (1:500; Beyotime Institute of Biotechnology, China). The membranes were then incubated with a goat anti-rabbit (no. A0208) or anti-mouse (no. A0216) IgG-conjugated to alkaline phosphatase secondary antibody for $2 \mathrm{~h}$. Finally, the membranes were washed 3 times with TBST and imaged using a gel imaging system (Bio-Rad, USA).
Statistical analysis. All data are represented as the mean \pm standard deviation (SD) of 3 repeated experiments. The Student's test was used to for comparison between two groups. $\mathrm{P}<0.05$ was considered statistically significant. All data were analyzed with SPSS statistical software 18.0.

\section{Results}

TALEN-mediated diallelic Nanog mutations in the HepG2 cells. To detect whether TALEN successfully achieved cleavage of the target site of the Nanog gene (Fig. 1A), genomic DNA from Nanog-targeted and control cells was extracted and then used to amplify the DNA fragment containing the Nanogtargeted site. The amplified product was analyzed by T7E1 to detect the activity and efficiency of TALEN. Surprisingly, the efficiency of the TALEN-mediated Nanog gene mutation approached $50 \%$ after two transfections (Fig. 1B). Subsequently, we selected out single cells for further culture from the Nanog-targeted mixture of cells. After 15 days, single cell-derived subclone genomes were extracted and analyzed by T7E1. Eventually, we identified subclones for further genomic 
A

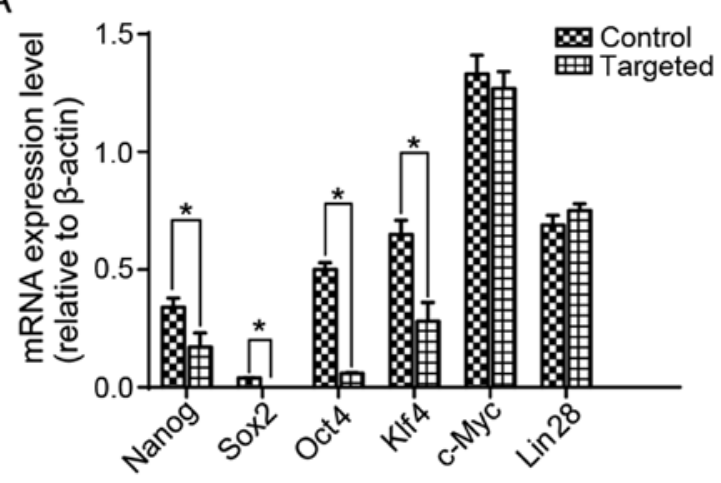

B

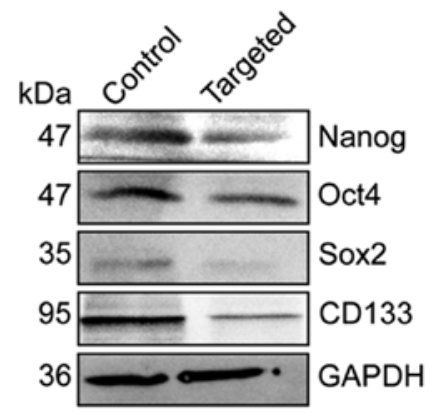

Figure 2. Effects of Nanog disruption on pluripotency genes and cancer stem cell markers. The data are presented as the mean \pm SD of 3 independent experiments. (A) The mRNA expression levels of pluripotency genes in the Nanog-targeted and control cells were detected by quantitative PCR; ${ }^{\text {P }<0.05 . ~(B) ~ T h e ~}$ protein expression levels of the core pluripotency genes Oct4, Sox 2 and Nanog, as well as the cancer stem cell marker CD133 in the Nanog-targeted and control cells.
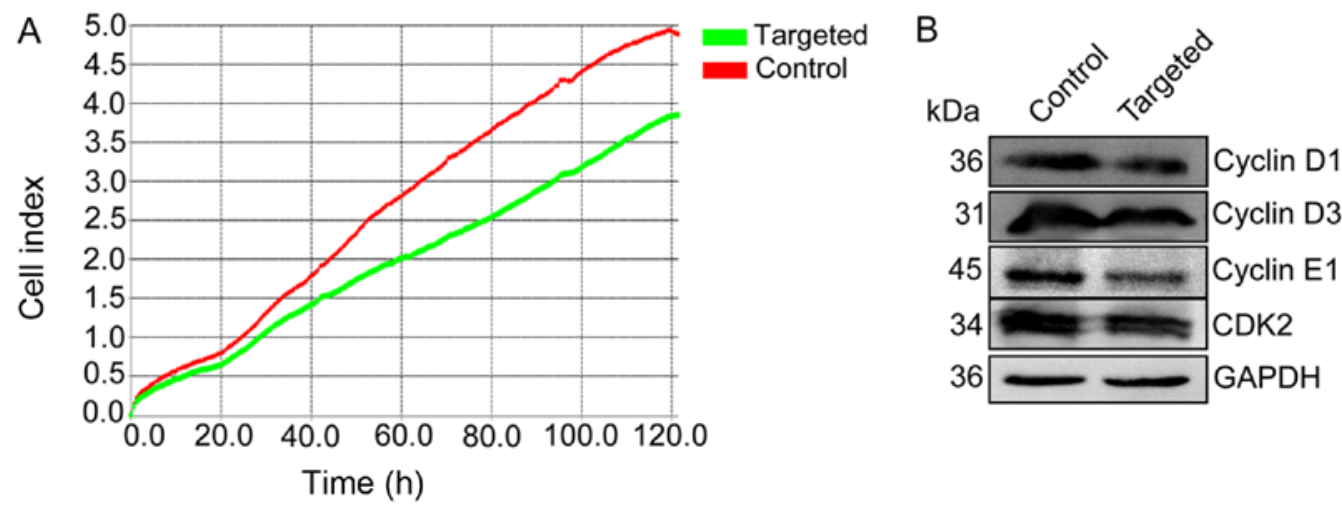

Figure 3. Effects of Nanog disruption on proliferative ability. (A) Proliferation curves of the Nanog-targeted and control cells as detected by an RTCA SP system over $120 \mathrm{~h}$. (B) The protein expression levels of proliferative markers in the control and Nanog-targeted cells.

sequencing, and found that subclone 3 and 7 exhibited biallelic Nanog mutations. The sequencing results indicated there were at least 3 Nanog alleles in the HepG2 cells (Fig. 1C).

Nanog regulates expression of pluripotency factors to maintain the pluripotency and malignancy of HepG2 cells. To determine the effects of Nanog disruption on stemness factors, we detected the expression of the pluripotency factors Oct4, Sox2, Klf4, c-Myc and Lin28, as well as the expression of cancer stem cell (CSC) marker CD133 by quantitative RT-PCR and western blotting. Our results indicated that the expression of Nanog, Oct4, Sox2, K1f4 and CD133 was markedly downregulated in the targeted cells, whereas the expression of c-Myc and Lin28 was not significantly different (Fig. 2A and B).

Disruption of Nanog suppresses the expression of proliferative markers in the HepG2 cells. To determine the effect of Nanog disruption on the apoptosis and proliferation of HepG2 cells, the proliferative capacity of Nanog-targeted and control cells was measured using an RTCA SP system, which demonstrated that the proliferative capacity of Nanog-targeted cells was markedly reduced relative to the control cells (Fig. 3A). We further detected cyclin D1/D3/E1 and cyclin-dependent kinase 2 (CDK2) protein expression by western blotting. In the Nanog-targeted cells, the expression levels of cyclin D1/D3/E1 and CDK2 protein were significantly reduced (Fig. 3B).

Disruption of Nanog impairs the migration and invasion of the HepG2 cells. The scratch assays indicated that Nanog disruption contributed to the reduced migration of HepG2 cells. As depicted in Fig. 4A, the migration distance of the targeted cells was significantly less than that of the control cells. CXCR4 is a migration-related factor that is able to induce tumor cell migration $(14,27,28)$. Notably, in the present study, we also found that CXCR4 expression was significantly decreased in the Nanog-targeted cells (Fig. 4B). Transwell assays indicated that the number of targeted cells that invaded through the membrane within $48 \mathrm{~h}$ was $15 \pm 5$, which was less than that of the control cells $(80 \pm 8 ; \mathrm{P}<0.01)$ (Fig. 4C). Previous studies have reported that matrix metallopeptidase 2 (MMP2) is an invasion-related gene that can regulate the invasive ability of tumor cells. High expression levels of MMP2 are closely related to tumor progression, invasion, metastasis and poor prognosis in various types of cancers $(14,29)$. In the present study, we also found that MMP2 expression was noticeably reduced in the targeted cells compared with expression in the control cells (Fig. 4D; $\mathrm{P}<0.05$ ). 
A

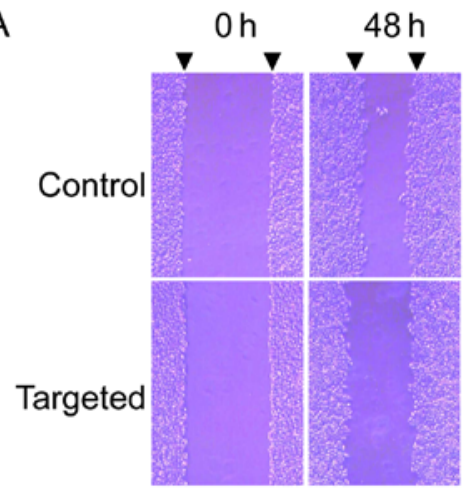

B
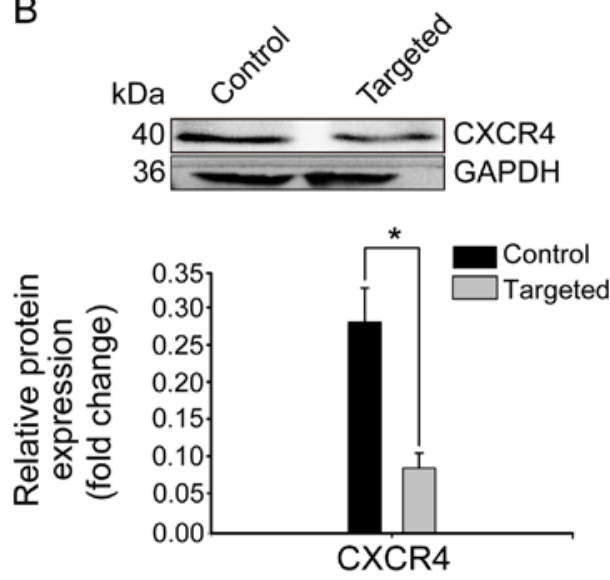
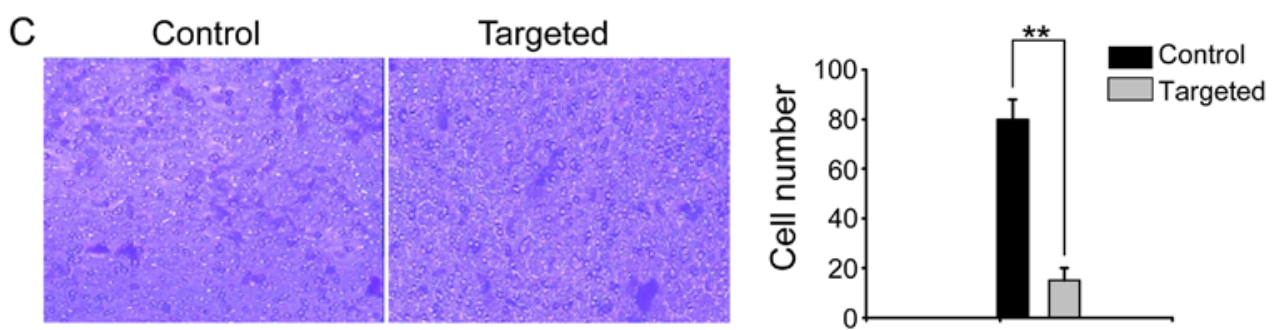

D
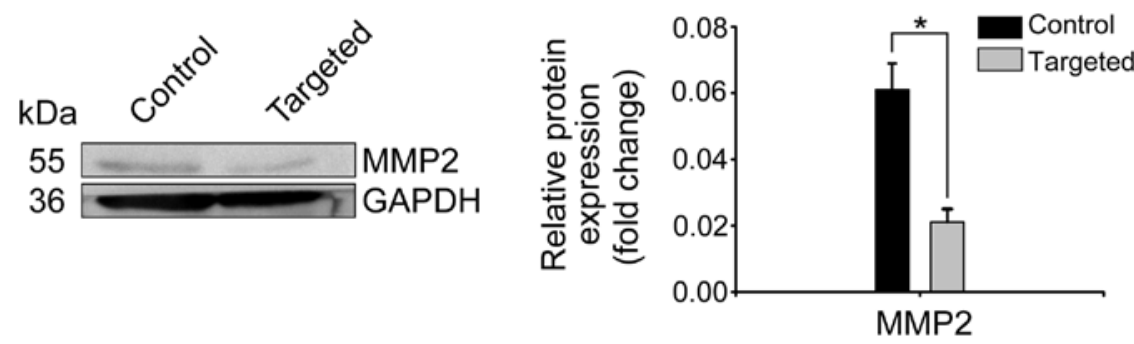

Figure 4. Nanog-mediated regulation of invasion and migration in the HepG2 cells. The data are presented as the mean \pm SD of 3 independent experiments. (A) Scratch assays were performed to assess the migratory capacity of the control and Nanog-targeted cells within $48 \mathrm{~h}$. (B) The expression levels of CXCR4 were determined by western blotting; ${ }^{*} \mathrm{P}<0.05$. (C) The migrated cells on the bottom side of the membrane were stained with $0.1 \%$ crystal violet; ${ }^{* *} \mathrm{P}<0.01$. (D) The expression level of MMP2 was determined by western blotting; ${ }^{\mathrm{P}}<0.05$.

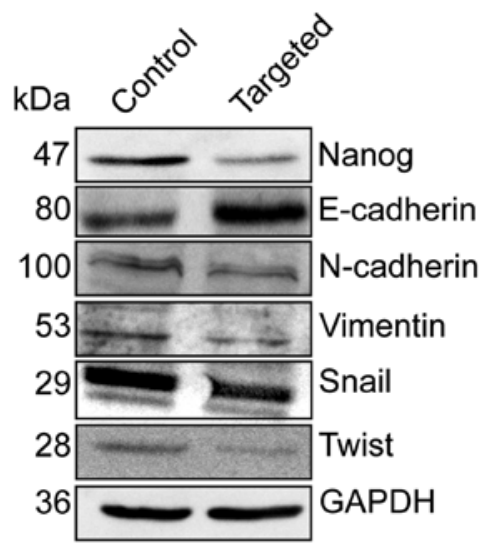

Figure 5. Nanog regulates EMT via the modulation of EMT-related regulators. The expression levels of EMT markers and EMT-related regulators were detected by western blotting.

Disruption of Nanog expression decreases EMT by downregulating EMT regulators Twist and Snail. To elucidate the effect of the disruption of Nanog on EMT, we detected the expression of the epithelial marker E-cadherin, and the mesenchymal markers $\mathrm{N}$-cadherin and vimentin by western blotting. Relative to the control cells, E-cadherin expression was elevated, while $\mathrm{N}$-cadherin and vimentin expression was decreased in the Nanog-targeted cells. We further detected the expression of the EMT regulators Twist and Snail, and found that their expression levels were decreased in the Nanogtargeted cells. (Fig. 5).

Disruption of Nanog enhances the chemosensitivity of HepG2 cells by downregulating multidrug resistance genes. To assess the effect of chemotherapeutics on HepG2 cells, both Nanogtargeted and control cells were exposed to cisplatin $(40 \mu \mathrm{g} /$ $\mathrm{ml}$ ), and chemosensitivity was measured using an RTCA SP system. After $72 \mathrm{~h}$ of observation, we noted that the Nanogtargeted cells were more sensitive to cisplatin than the control cells (Fig. 6A). Furthermore, we detected the expression of multi-drug resistant gene 1 (MDR1) and ATP-binding cassette subfamily G member 2 (ABCG2) in the Nanog-targeted and control cells, and found that expression levels of both were significantly downregulated in the Nanog-targeted cells (Fig. 6B). 
A

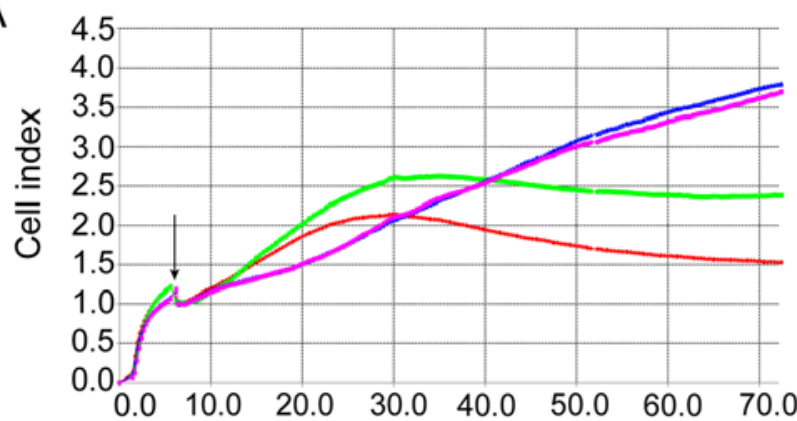

- Control - Targeted - Control+Cisplatin - Targeted + Cisplatin
B

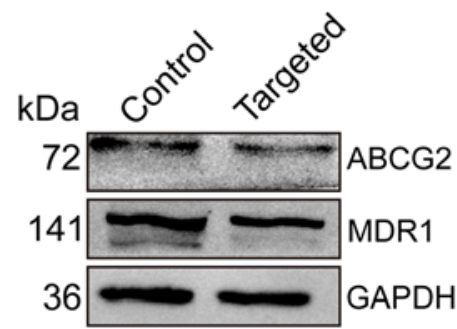

Time (h)

Figure 6. Chemosensitivity of the control and Nanog-targeted cells to chemotherapeutics. (A) Chemosensitivity of the control and Nanog-targeted cells to cisplatin $(40 \mu \mathrm{g} / \mathrm{ml})$ was determined by an RTCA SP system after $72 \mathrm{~h}$. Addition of cisplatin is indicated by a black vertical arrow. (B) The protein expression levels of multi-drug resistance genes ABCG2 and MDR1 relative to GAPDH.

\section{Discussion}

Increasing evidence suggests that Nanog expression is elevated in HCC cell lines and in primary tumors, and high Nanog expression is positively associated with HCC patient prognosis $(1,2,30)$. However, currently published investigations have failed to establish the complete underlying molecular mechanisms of Nanog in HepG2 cells. In the present study, we aimed to clarify the precise mechanisms by which Nanog affects the malignant behavior of the HCC HepG2 cell line.

Previous investigations have verified that Nanog is able to regulate cell cycle- and apoptosis-related factors to modulate proliferation and apoptosis in various types of cancers $(12,31)$. However, whether Nanog exhibits this function in HepG2 cells remained unclear. In the present study, we used an RTCA SP system to detect the proliferative ability of Nanog-targeted and control cells, and demonstrated for the first time that Nanog can regulate cyclin D1/D3/E and CDK2 to influence proliferation in HepG2 cells.

To investigate the effect of the disruption of Nanog on biological behavior, we conducted cell migration, Matrigel invasion and chemoresistance assays. Our data indicated that the disruption of Nanog confers an attenuated phenotype with respect to migration, invasion and chemoresistance. The migration-related gene CXCR4 and the invasion-related gene MMP2 play critical roles in migration and invasion in tumor cells, respectively $(28,29)$. Therefore, we also detected the expression levels of CXCR4 and MMP2 in Nanog-targeted and control cells, demonstrating that the disruption of Nanog reduced migration and invasion through downregulation of CXCR4 and MMP2 in the HepG2 cells, respectively. Therefore, inhibition of CXCR4 and MMP2 hindered HepG2 cell migration and invasion to a certain degree. The precise mechanisms by which Nanog regulates CXCR4 and MMP2 require further research, which may reveal novel therapies and strategies to suppress the migration and invasion of HCC. Chemoresistance is always a large barrier for completely eradicating tumors through anticancer drugs. In the present study, we also detected the sensitivity of Nanog-targeted and control cells to cisplatin ( $40 \mu \mathrm{g} / \mathrm{ml})$ using an RTCA SP system after $72 \mathrm{~h}$, and demonstrated that the disruption of Nanog rendered the HepG2 cells more sensitive to cisplatin $(40 \mu \mathrm{g} /$ $\mathrm{ml})$. We further detected the expression of ABCG2 and MDR1 in the Nanog-targeted and control cells; and revealed that the disruption of Nanog expression downregulated ABCG2 and MDR1 expression to induce chemosensitivity in the HepG2 cells, causing HepG2 cells to have increased sensitivity to chemotherapeutics. Our results suggest that targeting Nanog, along with the administration of conventional anticancer drugs, will be an optimal therapy with which to improve the prognosis and survival rate of HCC patients.

EMT plays a crucial role in promoting invasion and metastasis during tumor progression. During EMT, tumor cells reduce cell-cell adhesion to acquire a mesenchymal-like phenotype and disseminate into neighboring or distant tissues (32,33). Therefore, enhanced EMT disrupts E-cadherinmediated cell-cell adhesion and converts epithelial-like cells into mesenchymal-like cells during tumor cell progression. In the present study, we detected the EMT markers in Nanogtargeted and control cells, and found that the disruption of Nanog resulted in inhibition of the EMT process with an elevated E-cadherin level, and with decreased N-cadherin and vimentin levels in HepG2 cells. Intensive studies have revealed that transcription factors including Snail and Twist regulate the EMT process (34-36). In the present study, we further detected the expression levels of Snail and Twist in the Nanog-targeted and control cells, and demonstrating that the expression of these transcription factors also decreased. These data suggest that Nanog participates in the regulation of the EMT process to influence the metastasis of HepG2 cells via modulating Snail and Twist. Our data also indicated that the reversal of Snail and Twist expression may contribute to the restoration of EMT, which may be an alternative target for the future gene therapy of HCC patients to block the metastasis and dissemination of tumor cells.

\section{Acknowledgements}

The present study was supported by grants from the Ministry of Science and Technology of China (2013ZX10001004002-005), the Natural Science Foundation of Hubei Province of China (2013CFC033), and the Hubei University of Medicine Juvenile Scientific and Technological Creativity Team (2014 CXZ06). 


\section{References}

1. Shan J, Shen J, Liu L, Xia F, Xu C, Duan G, Xu Y, Ma Q, Yang Z, Zhang $Q$, et al: Nanog regulates self-renewal of cancer stem cells through the insulin-like growth factor pathway in human hepatocellular carcinoma. Hepatology 56: 1004-1014, 2012

2. Sun C, Sun L, Jiang K, Gao DM, Kang XN, Wang C, Zhang S, Huang S, Qin X, Li Y, et al: NANOG promotes liver cancer cell invasion by inducing epithelial-mesenchymal transition through NODAL/SMAD3 signaling pathway. Int J Biochem Cell Biol 45: 1099-1108, 2013.

3. Hashimoto N, Tsunedomi R, Yoshimura K, Watanabe Y, Hazama S and Oka M: Cancer stem-like sphere cells induced from de-differentiated hepatocellular carcinoma-derived cell lines possess the resistance to anti-cancer drugs. BMC Cancer 14: $722,2014$.

4. Li X, Luo Q and Song G: Novel therapeutic strategies for treatment of hepatocellular carcinoma: Targeting intervention on liver cancer stem cells. Sheng Wu Yi Xue Gong Cheng Xue Za Zhi 30: 894-898, 2013 (In Chinese).

5. Wang J, Levasseur DN and Orkin SH: Requirement of Nanog dimerization for stem cell self-renewal and pluripotency. Proc Natl Acad Sci USA 105: 6326-6331, 2008.

6. Pan G and Thomson JA: Nanog and transcriptional networks in embryonic stem cell pluripotency. Cell Res 17: 42-49, 2007.

7. Wang J, Rao S, Chu J, Shen X, Levasseur DN, Theunissen TW and Orkin SH: A protein interaction network for pluripotency of embryonic stem cells. Nature 444: 364-368, 2006.

8. Hyslop L, Stojkovic M, Armstrong L, Walter T, Stojkovic P, Przyborski S, Herbert M, Murdoch A, Strachan T and Lako M: Downregulation of NANOG induces differentiation of human embryonic stem cells to extraembryonic lineages. Stem Cells 23: 1035-1043, 2005.

9. Mitsui K, Tokuzawa Y, Itoh $\mathrm{H}$, Segawa K, Murakami M, Takahashi K, Maruyama M, Maeda M and Yamanaka S: The homeoprotein Nanog is required for maintenance of pluripotency in mouse epiblast and ES cells. Cell 113: 631-642, 2003

10. Chambers I, Colby D, Robertson M, Nichols J, Lee S, Tweedie S and Smith A: Functional expression cloning of Nanog, a pluripotency sustaining factor in embryonic stem cells. Cell 113 643-655, 2003

11. Wang ML, Chiou SH and Wu CW: Targeting cancer stem cells: Emerging role of Nanog transcription factor. Onco Targets Ther 6: 1207-1220, 2013.

12. Iv Santaliz-Ruiz LE, Xie X, Old M, Teknos TN and Pan Q: Emerging role of nanog in tumorigenesis and cancer stem cells. Int J Cancer 135: 2741-2748, 2014.

13. Yang L, Zhang X, Zhang M, Zhang J, Sheng Y, Sun X, Chen Q and Wang LX: Increased Nanog expression promotes tumor development and cisplatin resistance in human esophageal cancer cells. Cell Physiol Biochem 30: 943-952, 2012.

14. Lu Y, Zhu H, Shan H, Lu J, Chang X, Li X, Lu J, Fan X, Zhu S, Wang Y, et al: Knockdown of Oct4 and Nanog expression inhibits the stemness of pancreatic cancer cells. Cancer Lett 340: 113-123, 2013

15. Wang D, Lu P, Zhang H, Luo M, Zhang X, Wei X, Gao J, Zhao Z and Liu C: Oct-4 and Nanog promote the epithelial-mesenchymal transition of breast cancer stem cells and are associated with poor prognosis in breast cancer patients. Oncotarget 5: 10803-10815, 2014.

16. Huang CE, Yu CC, Hu FW, Chou MY and Tsai LL: Enhanced chemosensitivity by targeting Nanog in head and neck squamous cell carcinomas. Int J Mol Sci 15: 14935-14948, 2014.

17. Ding Y, Yu AQ, Li CL, Fang J, Zeng Y and Li DS: TALENmediated Nanog disruption results in less invasiveness, more chemosensitivity and reversal of EMT in Hela cells. Oncotarget 5 : 8393-8401, 2014.

18. Chanrion M, Kuperstein I, Barrière C, El Marjou F, Cohen D, Vignjevic D, Stimmer L, Paul-Gilloteaux P, Bièche I, Tavares SR, et al: Concomitant Notch activation and p53 deletion trigger epithelial-to-mesenchymal transition and metastasis in mouse gut. Nat Commun 5: 5005, 2014.
19. Rangel MC, Karasawa H, Castro NP, Nagaoka T, Salomon DS and Bianco C: Role of Cripto-1 during epithelial-to-mesenchymal transition in development and cancer. Am J Pathol 180: 2188-2200, 2012

20. Mani SA, Guo W, Liao MJ, Eaton EN, Ayyanan A, Zhou AY, Brooks M, Reinhard F, Zhang CC, Shipitsin M, et al: The epithelial-mesenchymal transition generates cells with properties of stem cells. Cell 133: 704-715, 2008

21. Polyak K and Weinberg RA: Transitions between epithelial and mesenchymal states: Acquisition of malignant and stem cell traits. Nat Rev Cancer 9: 265-273, 2009.

22. Thiery JP and Sleeman JP: Complex networks orchestrate epithelial-mesenchymal transitions. Nat Rev Mol Cell Biol 7: 131-142, 2006.

23. Katsuyama T, Akmammedov A, Seimiya M, Hess SC, Sievers C and Paro R: An efficient strategy for TALEN-mediated genome engineering in Drosophila. Nucleic Acids Res 41: e163, 2013.

24. Li T, Huang S, Zhao X, Wright DA, Carpenter S, Spalding MH, Weeks DP and Yang B: Modularly assembled designer TAL effector nucleases for targeted gene knockout and gene replacement in eukaryotes. Nucleic Acids Res 39: 6315-6325, 2011.

25. Zhu H, Lau CH, Goh SL, Liang Q, Chen C, Du S, Phang RZ, Tay FC, Tan WK, Li Z, et al: Baculoviral transduction facilitates TALEN-mediated targeted transgene integration and Cre/LoxP cassette exchange in human-induced pluripotent stem cells. Nucleic Acids Res 41: e180, 2013.

26. Jiang D, Zhu W, Wang Y, Sun C, Zhang KQ and Yang J: Molecular tools for functional genomics in filamentous fungi: Recent advances and new strategies. Biotechnol Adv 31: 1562-1574, 2013.

27. Kucia M, Jankowski K, Reca R, Wysoczynski M, Bandura L, Allendorf DJ, Zhang J, Ratajczak J and Ratajczak MZ: CXCR4SDF-1 signalling, locomotion, chemotaxis and adhesion. J Mol Histol 35: 233-245, 2004

28. Müller A, Homey B, Soto H, Ge N, Catron D, Buchanan ME, McClanahan T, Murphy E, Yuan W, Wagner SN, et al: Involvement of chemokine receptors in breast cancer metastasis. Nature 410: 50-56, 2001

29. Egeblad M and Werb Z: New functions for the matrix metalloproteinases in cancer progression. Nat Rev Cancer 2: 161-174, 2002.

30. Wang XQ, Ng RK, Ming X, Zhang W, Chen L, Chu AC, Pang R, Lo CM, Tsao SW, Liu X, et al: Epigenetic regulation of pluripotent genes mediates stem cell features in human hepatocellular carcinoma and cancer cell lines. PLoS One 8: e72435, 2013.

31. Han J, Zhang F, Yu M, Zhao P, Ji W, Zhang H, Wu B, Wang Y and Niu R: RNA interference-mediated silencing of NANOG reduces cell proliferation and induces G0/G1 cell cycle arrest in breast cancer cells. Cancer Lett 321: 80-88, 2012.

32. Casas E, Kim J, Bendesky A, Ohno-Machado L, Wolfe CJ and Yang J: Snail2 is an essential mediator of Twistl-induced epithelial mesenchymal transition and metastasis. Cancer Res 71: 245-254, 2011

33. Acloque H, Adams MS, Fishwick K, Bronner-Fraser M and Nieto MA: Epithelial-mesenchymal transitions: The importance of changing cell state in development and disease. J Clin Invest 119: 1438-1449, 2009.

34. Chiou SH, Wang ML, Chou YT, Chen CJ, Hong CF, Hsieh WJ, Chang HT, Chen YS, Lin TW, Hsu HS, et al: Coexpression of Oct4 and Nanog enhances malignancy in lung adenocarcinoma by inducing cancer stem cell-like properties and epithelialmesenchymal transdifferentiation. Cancer Res 70: 10433-10444, 2010.

35. Valastyan S and Weinberg RA: Tumor metastasis: Molecular insights and evolving paradigms. Cell 147: 275-292, 2011.

36. Meng HM, Zheng P, Wang XY, Liu C, Sui HM, Wu SJ, Zhou J, Ding YQ and Li J: Over-expression of Nanog predicts tumor progression and poor prognosis in colorectal cancer. Cancer Biol Ther 9: 295-302, 2010. 